\title{
EPOXY-LESS PACKAGING METHODS FOR ELECTRICAL CONTACT TO PARYLENE- BASED FLAT FLEXIBLE CABLES
}

\author{
Christian A. Gutierrez, Curtis Lee, Brian Kim and Ellis Meng \\ University of Southern California, Department of Biomedical Engineering, Los Angeles, CA, USA
}

\begin{abstract}
We present two methods for establishing rapid epoxyless electrical connectivity to Parylene-based flat flexible cables (FFC). The first utilizes commercially available zero-insertion-force (ZIF) connector technology and the second utilizes a custom-fabricated connector with an acrylic/polydimethylsiloxane (PDMS) interface featuring screen-printed contacts. A contact pitch of $0.5 \mathrm{~mm}$ was achieved using both connector methods. These techniques are simple to implement, reversible, scalable and unlike epoxy-based approaches, do not require manual intervention to secure individual contacts.
\end{abstract}

\section{KEYWORDS}

Parylene C, flat flexible cable (FFC), zero-insertionforce (ZIF), screen printing.

\section{BACKGROUND}

Parylene has emerged as a popular substrate for microelectrode technology due to its biocompatibility and mechanical strength (Young's modulus $E \sim 4 \mathrm{GPa}$ ). Compared to traditional FFC materials (e.g. polyimide, $E$ $\sim 7.5 \mathrm{GPa}$ ), Parylene is considerably softer, thus achieving robust electrical contacts to Parylene-based devices is a challenge. The use of thin-film metallization, as is often the case in Parylene FFCs, also makes them susceptible to scratching/delamination and requires care when establishing connections. The combination of these challenges and a tendency to place a secondary emphasis on connectivity/packaging often results in methods that largely employ manual application of conductive epoxies to secure connections [1-3]. Although these methods can be effective, they limit the ability to scale experiments, can be time intensive, can introduce error and/or noise and for most practical applications, are not a viable solution. Here, we present two epoxy-less methods for establishing simultaneous and reversible electrical connections to Parylene-based FFCs.

\section{METHODS AND FABRICATION}

\section{Parylene FFC Fabrication}

Parylene FFCs consisting of 8,18 and 30 contacts were designed for characterization purposes. One end of the FFCs includes contact pads designed to interface with either a (1) ZIF or (2) custom connector, while the other end comprised open electrode sites for continuity checking and cable resistance measurements (Fig. 1). The FFCs were fabricated by first coating a standard silicon wafer with Parylene-C (10-14 $\mu \mathrm{m})$. Thin-film platinum $(2000 \AA)$ was then deposited in an electron-beam

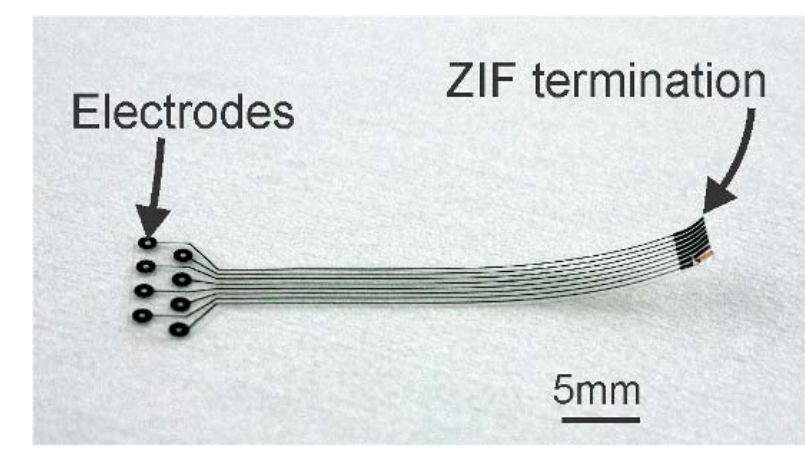

Figure 1: 8-channel Parylene FFC with ZIF-style termination

evaporator and patterned by lift-off. A second insulating layer of Parylene was then deposited $(2 \mu \mathrm{m})$ and patterned by reactive ion etching (RIE) in oxygen plasma to expose contact pads and electrodes (100 W, $100 \mathrm{mT})$. The cables were then released by deep reactive ion etching (DRIE) of the cable perimeter using a switched chemistry process $[4]$.

\section{Method 1 - ZIF Interface}

A commercially available flip-lock ZIF connector (Hirose, FH12-8S-0.5SH, $0.5 \mathrm{~mm}$ pitch, bottom contact) was selected and consisted of raised gold-plated phosphor bronze contacts. Simple and rapid connections were established with this connector type by using its hinged actuator which closes and locks onto the cable for a secure, reversible connection (8 or 18 channel, Fig. 2).

Commercially available ZIF connectors require cables of a minimum thickness in order to achieve a proper and secure interface with FFCs. Significant deviations from the recommended thickness results in unreliable connections and may cause damage to the cable. This kind of connector is typically utilized with polyimide FFCs with thicknesses generally greater than $200 \mu \mathrm{m}$. In contrast to polyimide, Parylene is deposited in a roomtemperature chemical vapor deposition process with

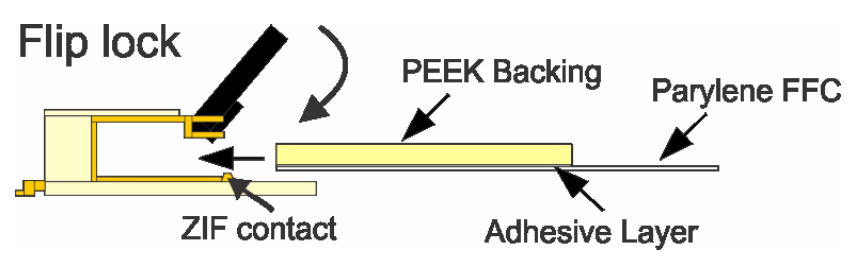

Figure 2: Profile view of flip-lock, bottom-contact, ZIF interconnect. 


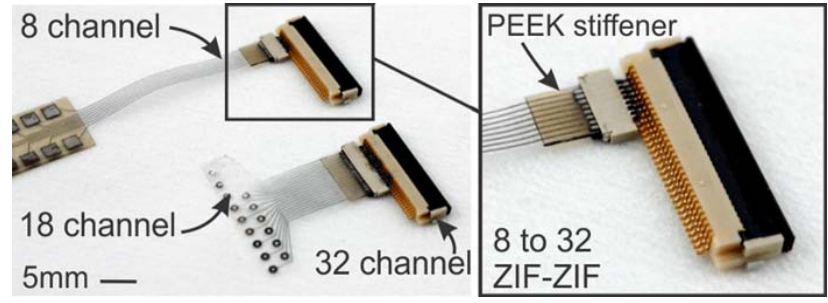

Figure 3: Parylene FFC-ZIF connectors. 8 and 18-channel Parylene FFC cables were mated to 8 and 18-channel ZIF connectors which were adapted back-to-back with a 32channel ZIF connector for ZIF-to-ZIF implementation. ZIF connectors used standard surface mount contacts.

typical deposition thicknesses ranging from 1-20 $\mu \mathrm{m}$. The significantly thinner Parylene cables thus require an additional backplate or stiffener around the connection interface region as a means of providing greater thickness.

A PEEK (polyether-ether-ketone) backing $(254 \mu \mathrm{m}$ thick) was affixed by means of cyanoacrylate adhesive to stiffen the cable. The etched border of the Parylene cable was traced onto the PEEK using a razor blade to create the exact dimensions for insertion into the ZIF connector. The final thickness of the inserted composite cable was $320 \mu \mathrm{m}$.

Standard ZIF connectors of this size are typically utilized to interface FFC with circuit boards and as such generally feature surface-mount contacts. In the case of stand-alone Parylene-based FFC this may not be desirable as cable lengths will tend to be limited by substrates on which the Parylene FFCs are fabricated. Hence, we also demonstrate the ability to create a ZIF-toZIF adaptor by soldering two ZIF connectors back-toback. This provides the capability of interfacing and extending the generally short Parylene FFCs with a standard FFCs, (eg. polyimide), for added flexibility in implementation (Fig. 3).

\section{Method 2 - Custom Interface}

For applications that require complete flexibility in design, it is desirable to have a means of realizing a custom connection interface of arbitrary geometry to Parylene FFCs. This is especially useful in applications where, for example, contacts are placed around the perimeter of a Parylene film or if non-symmetric contact spacing is required. This custom interface was realized by compression of screen printed silver paste $(\sim 30 \mu \mathrm{m}$ thick) patterned on a compliant PDMS sheet $(\sim 500 \mu \mathrm{m}$ thick) onto contacts on the Parylene FFC (Fig. 4). The silver paste provides a conductive link between the FFC pads and the conductive leads which make contact with the paste via drilled holes in the acrylic plate. Unlike conventional ZIF connectors, the silver paste/PDMS composite provides a very compliant yet conductive contact surface for mating against the Parylene FFC. This reduces the risk of scratching or delamination of fragile thin-film metal contacts.

The FFC, silver paste and PDMS were sandwiched

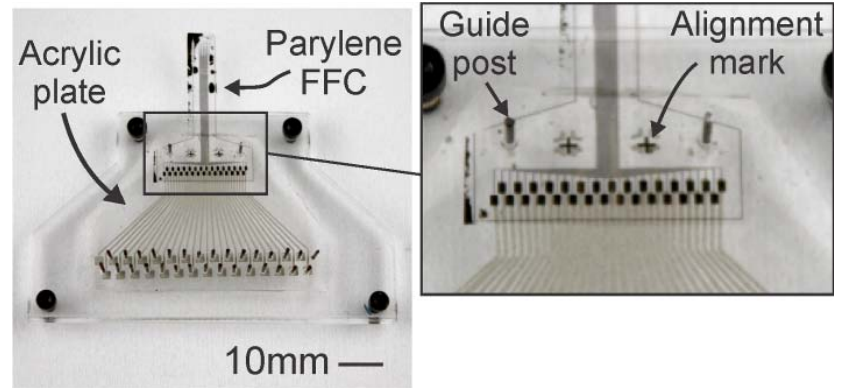

Figure 4: Parylene FFC (30-channel, $0.5 \mathrm{~mm}$ pitch) placed and clamped inside customer acrylic/PDMS connector. The photograph on the right shows a detail of the Parylene FFC and PDMS connector interface. Alignment marks and guide posts are shown.

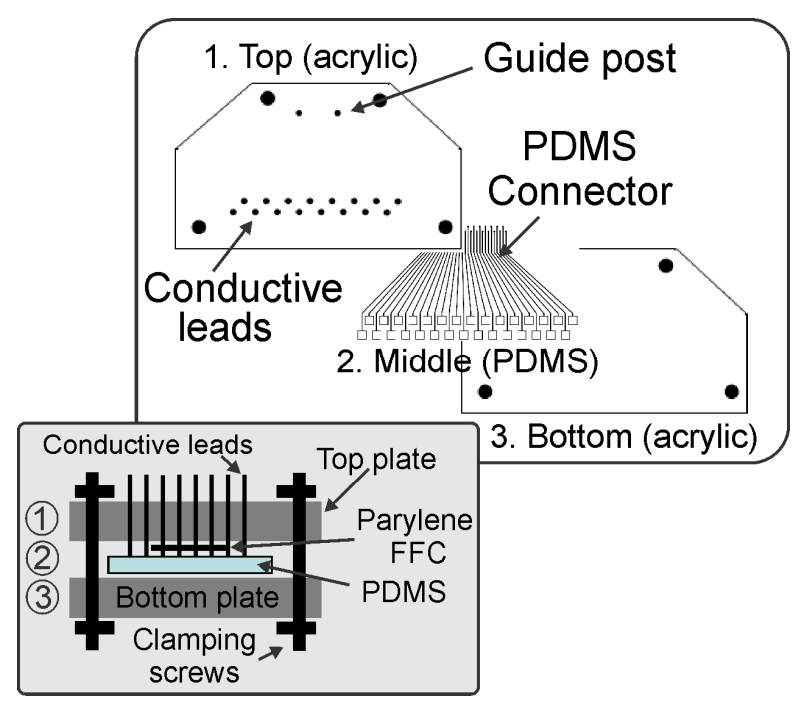

Figure 5: PDMS sheet with screen-printed silver-paste and Parylene cable within clamped acrylic assembly. The upper right shows the parts of the custom interface including the two acrylic plates and PDMS connector.

between two laser-machined acrylic plates and secured in place with screws. The screws also provide a means for controlling the amount of compression applied. Alignment of the connector with the FFC was accomplished through the use of matching alignment marks on the FCC and PDMS as well as guide posts integrated into the acrylic plates (Figs. 4 and 5).

A lithographically-patterned brass sheet was etched to produce a stencil for screen printing silver paste onto the PDMS sheet (Fig. 6). The brash sheet was secured by an acrylic frame and placed over the PDMS substrate for printing. A precision metal-blade squeegee was then utilized to press the silver paste through the openings in the brass sheet, thereby transferring the pattern onto the underlying PDMS substrate. The patterned paste was then cured in an oven prior to use $\left(130^{\circ} \mathrm{C}, 5\right.$ hours $)$. We have previously applied this method to the fabrication of PDMS-based carbon thick-film strain sensors and demonstrated a printing resolution down to $75 \mu \mathrm{m}$ line width using this technique [5]. 

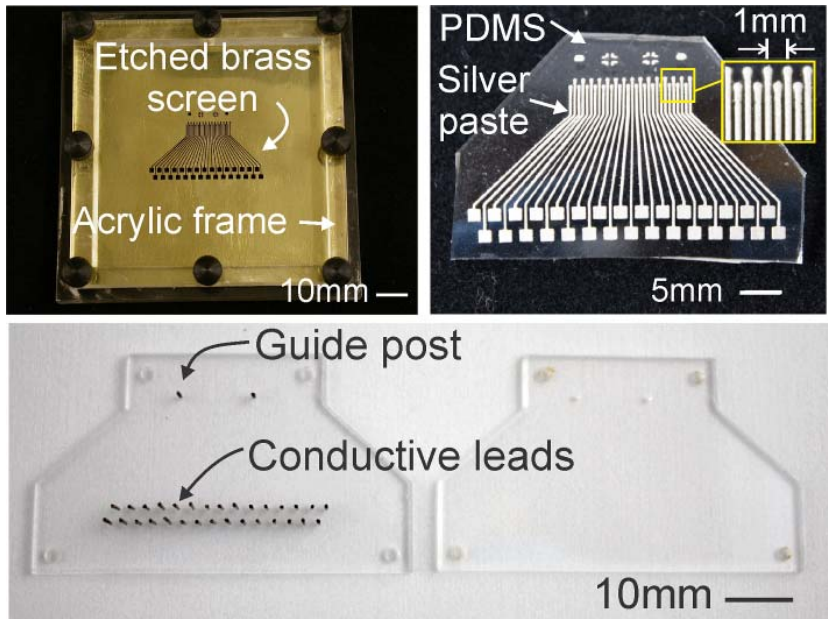

Figure 6: (Top): Etched brass screen used to screen-print silver paste onto PDMS sheet (top-right). (Bottom) lasermachined acrylic plates with alignment guide posts.

\section{RESULTS}

\section{Method 1 - ZIF Interface}

Electrical characterization of the interface was performed by measuring end-to-end cable resistance, optical observation of FCC contact pads and pull-out force measurement to assess connection robustness.

Successful connections were established across all contacts as verified by resistance measurements $(495 \pm 21.5 \Omega, \mathrm{n}=80)$. This verified that the composite cable/stiffener thickness and alignment within the ZIF connector were appropriate for establishing repeatable secure connections. The effect of cable orientation was also assessed by applying varying degrees of curvature to the Parylene FFC $\left(0^{\circ}, 90^{\circ}, 180^{\circ}, 360^{\circ}\right)$ (Fig. 7). No significant change to the in the average measured cable resistance was observed under any of these conditions.

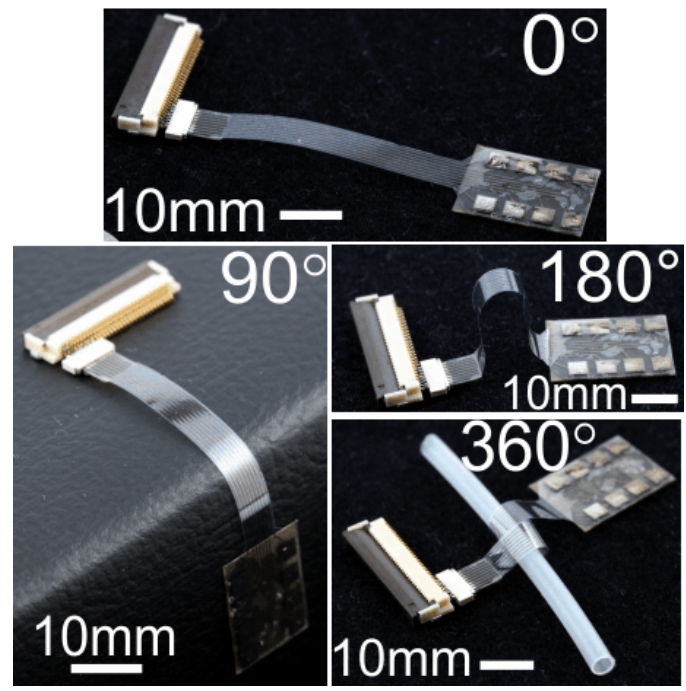

Figure 7: Cable orientations $\left(0^{\circ}, 90^{\circ}, 180^{\circ}, 360^{\circ}\right)$. Resistance measured across 4 channels. Maximum deviation of $0.7 \%$ among the different cable orientations.

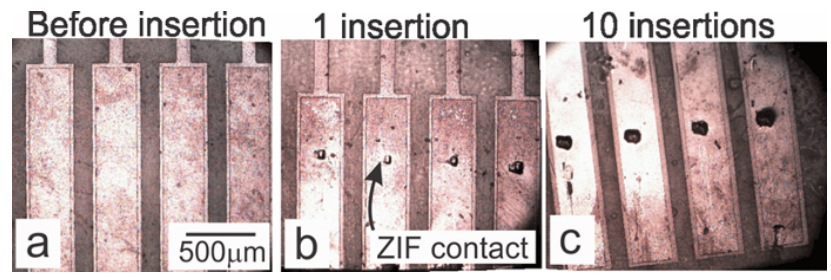

Figure 8: Optical micrographs of (a) Parylene cable pads prior to insertion, (b) Parylene cable pad after 1 insertion. [note: indentation due to ZIF connector], and (c) Parylene cable pad after 10 insertions: [note: deep indentations due to ZIF connector].

The effect of multiple insertions was assessed by repeating the insertion process (consisting of insertion , closure, release and removal) of the FFC ten times. The condition of the FFC contact pad was tracked optically to detect any mechanical deterioration. It was found that the Parylene cable (8-channel) maintained stable and repeatable connections over the ten cycles as evidenced by consistent resistance measurements; however clear depressions at contact points between the ZIF connector and Parylene cable were observed (Fig. 8).

A failure mode was identified after 15 insertion cycles (on a single FFC pad) due to mechanical damage from a raised contact point within the ZIF connector. Although all pads exhibited some wear at 15 cycles, the added fatigue induced by the raised contact point resulted in the early failure. This non-uniformity was likely due to excessive heating of the plastic housing during soldering causing the metal contact to shift within the ZIF assembly. Robustness of the connection was demonstrated in pull-out force measurements (Fig. 9). The ZIF assembly was fixed to a hard surface with a Parylene FFC inserted. A proof mass was then attached and hung vertically from the other end of the cable. The supported proof mass was incrementally increased until failure was observed. Failure of the cable occurred by fracture and ripping of the Parylene material and not at the ZIF connection interface. This was observed first for the 8-channel cable at a $120 \mathrm{~g}$ load followed by the 18 channel cable at $150 \mathrm{~g}$ load (Fig. 10). This failure mode occurred prior to cable pull-out, demonstrating the ability of ZIF connection interface to withstand significant loads.

\section{Method 2 - Custom Interface}

The lead resistance of a 30-channel Parylene FFC was measured from end tip to connector using micromanipulators connected to a multimeter. This resistance was typically $1 \mathrm{k} \Omega$ per lead. The average resistance of the silver paste leads, which is in-line with the cable resistance, was measured to be $15.75 \pm 6.66$ $\Omega,($ mean \pm SD, $\mathrm{n}=27)$ (Fig. 11). 


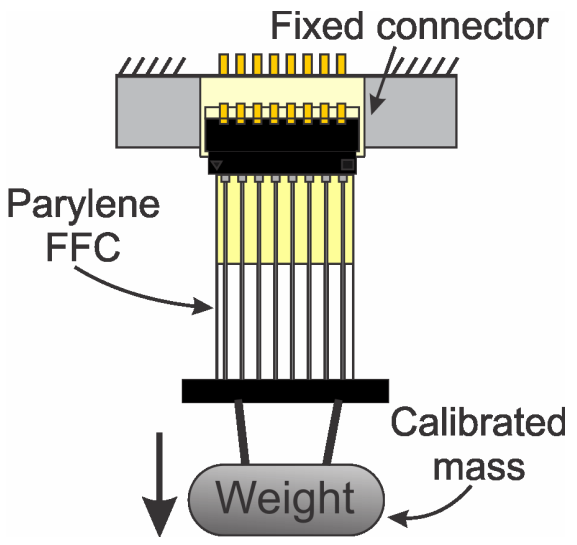

Figure 9: Experimental setup for pull-out force measurements.

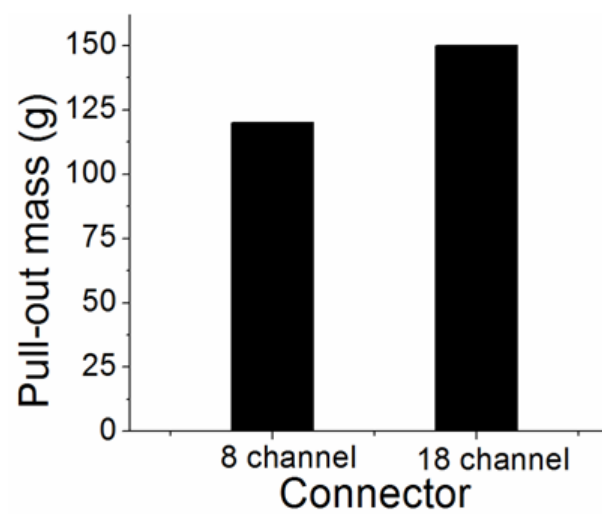

Figure 10: Maximum pull-out forces occurred prior to failure of Parylene FFC for both 8- and 18-channel connectors.

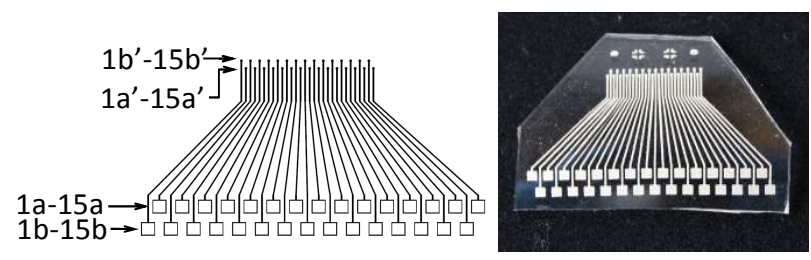

Figure 11: Layout of conductive silver paste leads. There are a total of 30 leads.

Once aligned, the PDMS slab was clamped and pressed onto the FFC contact pads and a robust connection was obtained. This procedure was repeated multiple times with no noticeable wear on the Pt pads. Conductive pins were inserted through holes in the acrylic layer and were used to make contact to enlarged silver pads on the PDMS. These pins enable direct measurement and provide the ability to solder wires or leads for downstream interfacing with the connector. Resistance measurements from pin to cable electrode were typically $1.3-1.4 \mathrm{k} \Omega$ which is in agreement measured values for each portion of the connector.

Alignment of the connector layers to the Parylene cable is the most critical step for this approach and is facilitated by the clear acrylic material and alignment guide posts. The alignment guide posts (which pass through the etched openings in the Parylene film) also ensure that the FFC lies securely within the connectors and prevents pull-out of the cable. The failure mode for this connector is expected to be ripping and/or fracture of the Parylene cable. It is also expected that this method will enable a high number of connection cycles without any noticeable wear to the FCC contact pads. A more thorough lifetime and fatigue study is required to validate this hypothesis.

\section{CONCLUSION}

The design, fabrication and characterization of two methods for establishing rapid epoxy-less electrical connectivity to Parylene-based flat flexible cables (FFC) was presented. Both the ZIF and custom acrylic/PDMS interface achieved a contact pitch of $0.5 \mathrm{~mm}$. ZIF connections were robust and outlasted the Parylene FFC in pull-out force measurements. These techniques are simple to implement, reversible, scalable and unlike epoxy-based approaches, do not require manual intervention to secure individual contacts.

\section{ACKNOWLEDGEMENTS}

This work was funded in part by Engineering Research Centers Program of the NSF under Number EEC-0310723, an NSF CAREER Award (ECS0547544), and the Bill and Melinda Gates Foundation (CG). The authors would like to thank Dr. Donghai Zhu and the members of the USC Biomedical Microsystems Laboratory for their assistance.

\section{REFERENCES:}

[1] J. Kitzmiller, D. Beversdorf, and D. Hansford, "Fabrication and testing of microelectrodes for smallfield cortical surface recordings," Biomed. Microdevices, vol. 8, pp. 81-85, 2006.

[2] C. Pang, S. Musallam, Y. C. Tai, J. W. Burdick, and R. A. Andersen, "Novel Monolithic Silicon Probes with Flexible Parylene Cables for Neural Prostheses," in Proc. Int. Conf. on Microtechnologies in Medicine and Biology, , 2006, pp. 64-67.

[3] B. Rubehn, C. Bosman, R. Oostenveld, P. Fries, and T. Stieglitz, "A MEMS-based flexible multichannel ECoG-electrode array," J. Neural Eng, vol. 6, pp. 10, 2009.

[4] E. Meng, P.-Y. Li, and Y.-C. Tai, "Plasma removal of Parylene C," J. Micromech. Microeng, vol. 18, pp. 045004, 2008.

[5] C. A. Gutierrez and E. Meng, "Low-cost carbon thick-film strain sensors for implantable applications," J. Micromech. Microeng, vol. 20, pp. 095028, 2010.

\section{CONTACT}

C.A. Gutierrez, tel: 213-821-3949; cagutier@usc.edu 